\title{
Effects of intravenous dofetilide on induction of atrioventricular re-entrant tachycardia
}

\author{
S M Cobbe, R W F Campbell, A J Camm, A W Nathan, E Rowland, P E Bloch-Thomsen, \\ M Møller, L Jordaens
}

\begin{abstract}
Objective-To assess the efficacy and safety of intravenous dofetilide in preventing induction of atrioventricular re-entrant tachycardia.

Design-A multicentre, open, dose ranging trial. Fifty one patients with electrically inducible atrioventricular re-entrant tachycardia were allocated to one of five doses of dofetilide $(1.5,3,6$, 9 , and $15 \mu \mathrm{g} / \mathrm{kg}$ ), two thirds of the dofetilide dose being given over a 15 minute loading period and the remainder over a 45 minute maintenance period.

Main outcome measure-Responders were defined as patients in whom dofetilide prevented reinduction of atrioventricular re-entrant tachycardia at the end of the infusion.

Results-Intravenous dofetilide had no effect on tachycardia inducibility at the two lower doses $(1.5$ and $3 \mu \mathrm{g} / \mathrm{kg})$ but prevented the reinduction of tachycardia at the three higher doses $(6,9$, and $15 \mu \mathrm{g} / \mathrm{kg})$ at a rate of $36 \%(11 / 31)$. There was a clear relation between plasma dofetilide concentrations and efficacy $(\mathrm{p}=0.009)$. In non-responders, dofetilide increased the cycle length of induced atrioventricular re-entrant tachycardia. Dofetilide increased the atrial and ventricular effective refractory periods, as well as the antegrade and retrograde effective refractory period of the accessory pathway. Treatment related side effects were reported in four patients, one with a new sustained incessant supraventricular tachycardia.
\end{abstract}

Department of Medical Cardiology, Glasgow Royal Infirmary, 10 Alexandra Parade, Glasgow G31 2ER, UK S M Cobbe

Department of Cardiology, The Freeman Hospital, Newcastle Upon Tyne, UK

R W F Campbell ${ }^{\star}$

Division of

Cardiological

Sciences, St George's

Hospital Medical

School, London SW17, UK

A J Camm

Cardiac Department, St Bartholomew's

Hospital, London EC1, UK

A W Nathan

Royal Brompton National Heart and Lung Hospitals, London SW3, UK E Rowland

Department of Cardiology, Gentofte Hospital, University of Copenhagen,

Copenhagen, Denmark

P E Bloch-Thomsen

Department of Cardiology B, Odense University Hospital,

Odense, Denmark M Møller

Department of Cardiology, State University Ghent Hospital, Ghent, Belgium

L Jordaens

Correspondence to: Professor Cobbe

stuart.cobbe@

clinmed.gla.ac.uk

Accepted 27 June 2001

*Deceased

Conclusions-Dofetilide shows promise as an agent for the prevention of atrioventricular re-entrant tachycardia in patients without structural heart disease.

(Heart 2001;86:522-526)

Keywords: atrioventricular re-entrant tachycardia; dofetilide; refractory period

Pharmacological treatment of supraventricular tachycardia remains a common approach despite the increased use of radiofrequency ablation. Atrioventricular re-entrant tachycardia is currently treated with various pharmacological agents. While class I antiarrhythmic drugs are effective in treating this form of tachycardia, there is a reluctance to use these agents, especially in patients with structural heart disease, because of potential proarrhythmia or undesirable side effects - as seen in the cardiac arrhythmia suppression trial (CAST). ${ }^{12}$ The most common class III antiarrhythmic agents (amiodarone and sotalol) are effective but also cause undesirable side effects. ${ }^{3-7}$ Calcium channel blockers have negative inotropic and chronotropic effects and are used less often. ${ }^{8}$

Dofetilide is a novel class III antiarrhythmic agent that produces potent and selective blockade of the rapidly activating component of the delayed rectifier current, $\mathrm{I}_{\mathrm{kr} \cdot}{ }^{9}{ }^{10}$ Studies have shown that dofetilide prolongs both atrial and ventricular monophasic action potential durations and increases atrial and ventricular effective and functional refractory periods. Because of its selective class III antiarrhythmic effect, dofetilide has no effect on atrioventricular conduction or sinus node function. ${ }^{11-13}$

Clinical reports have shown that dofetilide is effective in the treatment of supraventricular tachyarrhythmias, such as atrial fibrillation and atrial flutter. ${ }^{14-16}$ However, to date there have been no studies on the dose dependent effects of dofetilide on atrioventricular re-entrant tachycardia. Our goal in this study was to assess the efficacy of intravenous dofetilide, in five different dosing regimens, for treating patients with inducible atrioventricular re-entrant tachycardia, by determining its ability to prevent reinduction of the tachycardia.

\section{Methods}

STUDY DESIGN

An open, dose ranging study was designed to evaluate the efficacy and safety of dofetilide. Patients were recruited from eight centres. Patients with suspected supraventricular or ventricular tachyarrhythmias, or both, were referred for a routine electrophysiological evaluation. The patient was included in the study only if the results of the electrophysiological test were compatible with inducible atrioventricular re-entrant tachycardia.

Patients were assigned to one of five doses of dofetilide $(1.5,3,6,9$, and $15 \mu \mathrm{g} / \mathrm{kg})$. The total dofetilide dose was given over a one hour period, two thirds of the dose being given in an initial 15 minute loading period and the remainder over a final 45 minute maintenance period.

\section{PATIENTS}

At baseline, all patients underwent standard electrophysiological evaluation and programmed electrical stimulation to evaluate atrioventricular re-entrant tachycardia. Only patients aged 18-75 years with inducible atrioventricular re-entrant tachycardia at baseline were included in the study. Female subjects 
Table 1 Exclusion criteria

- Recent (< 6 weeks) myocardial infarction or unstable angina

- Supraventricular tachycardia other than atrioventricular re-entrant tachycardia

- Ventricular tachycardia at prestudy evaluation or at baseline electrophysiological evaluation

- Left ventricular ejection fraction $<20 \%$

- Antiarrhythmic treatment or use of any other drugs that prolong the QTc interval taken within five half lives of start of study

- Amiodarone treatment taken within 4 weeks of start of the study or a serum amiodarone concentration of $>0.4 \mathrm{mg} / 1$

- Resting QTc interval $>440 \mathrm{~ms}$ in a drug-free state and in the absence of pre-excitation or bundle branch block

- Resting supine diastolic blood pressure $>110 \mathrm{~mm} \mathrm{Hg}$

- Resting supine systolic blood pressure $<90$ or $>200 \mathrm{~mm} \mathrm{Hg}$

had to be of non-childbearing potential or using appropriate contraception. Before inclusion, each patient had a full clinical examination, a 12 lead ECG, and 24 hour Holter monitoring. Exclusion criteria are listed in table 1.

The protocol was approved by the local ethics committees and the study was monitored in accordance with standard operating procedures and good clinical practice. All patients provided written informed consent before entering the study, which was conducted in compliance with the 1989 Declaration of Helsinki.

\section{ELECTROPHYSIOLOGY STUDY}

A 12 lead ECG was recorded at the following times: at rest at the prestudy examination; during the baseline period; at $7.5,15,20,30,40$, $50,60,65$, and 75 minutes after the start of drug infusion; and at $1,2,4,8,10$, and 24 hours after completion of the maintenance infusion.

For arrhythmia induction and intracardiac recording, multipolar electrode catheters were inserted transvenously and positioned under fluoroscopy in the high lateral right atrium, in the right ventricle, and across the tricuspid valve in the region of the bundle of His; as needed, a multipolar catheter was placed in the coronary sinus. Standard surface ECG leads were displayed and recorded at the same time as the intracardiac recordings on a multichannel electrophysiological recorder at paper speeds of $100-250 \mathrm{~mm} / \mathrm{s}$. Body surface and intracardiac recordings were filtered at the appropriate frequencies. Atrial and ventricular pacing was applied at twice the diastolic threshold, using $2 \mathrm{~ms}$ rectangular pulses.

During the baseline period (lasting around 60 minutes), standard programmed electrical stimulation protocols were used to measure refractory periods and to attempt induction of atrioventricular re-entrant tachycardia.

The effective refractory period of the atrium was determined using the extrastimulus method, in which an eight beat train of paced atrial beats $\left(A_{1}\right)$, at basic cycle lengths of 400 and $600 \mathrm{~ms}$, was followed by a single premature stimulus $\left(\mathrm{A}_{2}\right)$. The atrial effective refractory period was defined as the longest $A_{1}-A_{2}$ interval that failed to result in an atrial depolarisation. The effective refractory period of the right ventricle was also determined, using the same extrastimulus technique as for the atrium. The ventricular effective refractory period was defined as the longest $\mathrm{V}_{1}-\mathrm{V}_{2}$ interval that failed to result in premature ventricular depolarisation.

The antegrade and retrograde effective refractory periods of the accessory pathway were also determined using the extrastimulus method. The antegrade effective refractory period was defined as the longest premature $A_{1}-A_{2}$ interval that did not result in ventricular pre-excitation, as manifested by loss of the delta wave in the surface QRS.

The retrograde effective refractory period was defined as the longest premature $V_{1}-V_{2}$ interval that did not result in atrial preexcitation, as manifested by normalisation of the retrograde atrial activation sequence.

Single and double atrial extrastimuli at cycle lengths of 600 and $400 \mathrm{~ms}$ were given in an attempt to induce orthodromic reciprocating tachycardia. When ventricular to atrial conduction was present, single ventricular extrastimuli at cycle lengths of 600 and $400 \mathrm{~ms}$ were also applied.

The tachyarrhythmia was defined as noninducible if $\leqslant 4$ non-stimulated ventricular beats were produced by the above protocol, inducible if $\geqslant 5$ non-stimulated ventricular beats at a frequency $>120$ beats/min were produced, and sustained if an inducible arrhythmia lasting $>30$ seconds was produced. Once the loading infusions and maintenance dose were completed, programmed electrical stimulation was repeated in an attempt to reinduce atrioventricular re-entrant tachycardia at the end of the maintenance infusion.

Following the electrophysiology study, patients were monitored over the next 24 hours with a Holter recorder.

PLASMA CONCENTRATIONS OF DOFETILIDE

Blood samples were obtained at $7.5,15,20,30$, $40,50,60,65$, and 75 minutes after the start of drug infusion. Blood plasma samples were stored at $-20^{\circ} \mathrm{C}$ and later analysed using radioimmunoassay techniques. ${ }^{17}$ The maximum plasma concentration, $\mathrm{C}_{\max }$, and the area under the nine hour dofetilide plasma concentration $v$ time curve, $\mathrm{AUC}_{0-9}$, were calculated for each subject.

\section{MEASUREMENTS}

PR interval and QRS durations were determined from the ECG recordings. The QT interval was measured from the same lead $\left(\mathrm{V}_{2}\right.$ if possible) by standard methods, and Bazett's formula was used to calculate QTc $\left(\mathrm{QTc}=\mathrm{QT} /[60 / \mathrm{HR}]^{1 / 2}\right.$, where QT is measured in milliseconds and heart rate is measured in beats/min).

The efficacy of dofetilide was determined by its ability to prevent the reinduction of an atrioventricular re-entrant tachycardia originally induced at baseline. Responders to dofetilide were defined as patients in whom this tachycardia could not be reinduced; nonresponders were defined as those in whom the tachycardia could be reinduced. In nonresponders, the cycle lengths of the tachycardia were compared before and after intravenous 


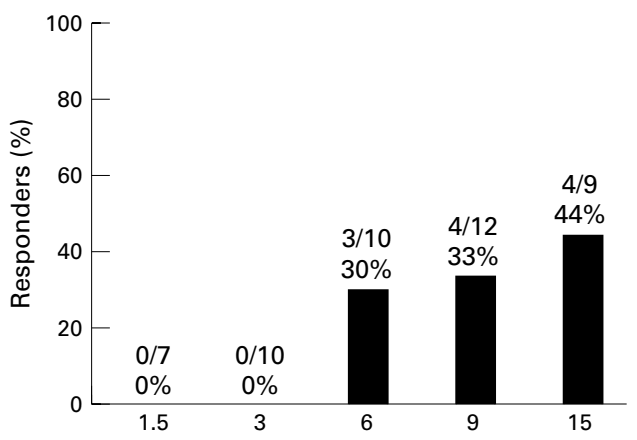

Figure 1 Percentage of patients responding to intravenous dofetilide at doses of $1.5,3,6,9$, and $15 \mu \mathrm{g} / \mathrm{kg}$.

Table 2 Atrioventricular re-entrant tachycardia response characteristics relative to dofetilide $A U C_{0-9}$

\begin{tabular}{ll}
\hline$A U C_{0-9}(n g . h / m l)$ & Number of responders (\%) \\
\hline 0 to $<5$ & $0 / 9(0)$ \\
5 to $<10$ & $0 / 7(0)$ \\
10 to $<15$ & $1 / 6(17)$ \\
15 to $<20$ & $0 / 7(0)$ \\
20 to $<25$ & $2 / 3(67)$ \\
25 & $2 / 4(50)$ \\
\hline
\end{tabular}

$\mathrm{AUC}_{0-9}$, area under 9 hour dofetilide plasma concentration $v$ time curve.

Table 3 Baseline tachycardia cycle length and changes from baseline by treatment group in non-responders

\begin{tabular}{lllll}
\hline $\begin{array}{l}\text { Dofetilide dose } \\
(\mu \mathrm{g} / \mathrm{kg})\end{array}$ & $n$ & $\begin{array}{l}\text { Baseline } \\
(\mathrm{ms})\end{array}$ & $n$ & $\begin{array}{l}\text { Change from } \\
\text { baseline }(\mathrm{ms})\end{array}$ \\
\hline 1.5 & 6 & $334(21)$ & 6 & $-2(15)$ \\
3.0 & 6 & $260(30)$ & 5 & $24(13)$ \\
6.0 & 7 & $349(15)$ & 7 & $31(11)$ \\
9.0 & 8 & $336(13)$ & 7 & $47(12)$ \\
15.0 & 5 & $386(37)$ & 4 & $9(7)$ \\
\hline
\end{tabular}

Values are mean (SEM).

infusion of dofetilide. The effective refractory periods of the atrium, ventricle, and accessory pathways were obtained during the baseline and dofetilide periods.

Table 4 Effective refractory periods and changes from baseline by treatment group

\begin{tabular}{|c|c|c|c|c|c|c|c|c|}
\hline \multirow{3}{*}{$\begin{array}{l}\text { Treatment (total } \\
\text { dose dofetilide } \\
(\mu \mathrm{g} / \mathrm{kg}))\end{array}$} & \multicolumn{8}{|c|}{ Cycle length } \\
\hline & \multicolumn{4}{|c|}{$400 \mathrm{~ms}$} & \multicolumn{4}{|c|}{$600 \mathrm{~ms}$} \\
\hline & $n$ & $\begin{array}{l}\text { Baseline } \\
(m s)\end{array}$ & $n$ & $\begin{array}{l}\text { Change from } \\
\text { baseline ( } \mathrm{ms} \text { ) }\end{array}$ & $n$ & $\begin{array}{l}\text { Baseline } \\
\text { (ms) }\end{array}$ & $n$ & $\begin{array}{l}\text { Change from } \\
\text { baseline (ms) }\end{array}$ \\
\hline \multicolumn{9}{|l|}{ Atrial } \\
\hline 1.5 & 5 & $192(15)$ & 3 & $40(31)$ & 5 & $208(12)$ & 5 & $11(2)$ \\
\hline 3.0 & 9 & $193(8)$ & 7 & $-7(11)$ & 9 & $199(5)$ & 9 & $6(8)$ \\
\hline 6.0 & 8 & $210(10)$ & 7 & $20(17)$ & 8 & $222(13)$ & 7 & $24(19)$ \\
\hline 9.0 & 9 & $189(9)$ & 6 & $28(11)$ & 5 & $223(12)$ & 5 & $9(15)$ \\
\hline 15.0 & 7 & $201(9)$ & 6 & $24(4)$ & 5 & $212(9)$ & 4 & $26(8)$ \\
\hline \multicolumn{9}{|l|}{ Ventricular } \\
\hline 1.5 & 5 & $211(8)$ & 5 & $4(12)$ & 6 & $226(6)$ & 6 & $6(13)$ \\
\hline 3.0 & 9 & $193(5)$ & 8 & $18(6)$ & 9 & $211(5)$ & 9 & $19(7)$ \\
\hline 6.0 & 9 & $201(7)$ & 9 & $20(7)$ & 8 & $227(9)$ & 8 & $20(12)$ \\
\hline 9.0 & 10 & $210(6)$ & 9 & $6(8)$ & 7 & $225(9)$ & 5 & $18(18)$ \\
\hline 15.0 & 7 & $196(4)$ & 6 & $38(7)$ & 6 & $218(5)$ & 5 & $35(5)$ \\
\hline \multicolumn{9}{|c|}{ Antegrade accessory pathway } \\
\hline 1.5 & 1 & 275 & 1 & 25 & 2 & $288(13)$ & 0 & \\
\hline 3.0 & 4 & 259 (13) & 2 & $-23(38)$ & 6 & $267(14)$ & 3 & $10(6)$ \\
\hline 6.0 & 6 & $270(14)$ & 3 & $22(2)$ & 5 & $352(49)$ & 4 & $15(21)$ \\
\hline 9.0 & 4 & $275(9)$ & 3 & $47(13)$ & 2 & $400(70)$ & 1 & 20 \\
\hline 15.0 & 5 & $276(11)$ & 2 & $35(5)$ & 3 & $303(38)$ & 3 & $70(6)$ \\
\hline \multicolumn{9}{|c|}{ Retrograde accessory pathway } \\
\hline 1.5 & 2 & $255(15)$ & 1 & 0 & 0 & & 0 & \\
\hline 3.0 & 5 & $221(13)$ & 4 & $36(9)$ & 4 & $245(4)$ & 3 & $65(30)$ \\
\hline 6.0 & 5 & $262(26)$ & 4 & $30(9)$ & 5 & $281(25)$ & 4 & $69(17)$ \\
\hline 9.0 & 2 & $285(35)$ & 2 & $95(25)$ & 2 & $265(25)$ & 1 & 120 \\
\hline 15.0 & 2 & $285(15)$ & 1 & 50 & 2 & $285(5)$ & 1 & 90 \\
\hline
\end{tabular}

Values are mean (SEM).
ANALYSIS

Response rates were compared using the Cochran-Mantel-Haenszel test to determine the relation between drug dose or plasma concentration and response. Data are reported as mean (SEM). A probability value of $\mathrm{p}<0.05$ was considered significant.

\section{Results}

PATIENTS

Of the 59 patients screened, 51 (41 men, 10 women) were enrolled to the study and 48 were followed to completion. All groups were well balanced with respect to age (mean 39 years; range 18-69 years) and weight (mean $76 \mathrm{~kg}$; range $55-110 \mathrm{~kg}$ ). Included in the safety but not the efficacy analysis were three patients who received doses not defined in the original protocol. There were no significant differences in PR interval, QRS duration, heart rate, or systemic blood pressure. Incomplete data prevented assessment of the prevalence and type of structural heart disease at baseline.

\section{EFFICACY}

Sustained atrioventricular re-entrant tachycardia was induced in all 51 patients before dofetilide infusion. The tachycardia was reinduced in all patients receiving dofetilide at the two lowest doses; however, it was suppressed in patients receiving dofetilide at the three higher doses. The response for each dofetilide dose group is shown in fig 1 . Dofetilide was effective in preventing atrioventricular re-entrant tachycardia in $36 \%$ of the patients $(11 / 31)$ in the three highest dose groups. The dose-response relation was significant $(\mathrm{p}=0.009)$.

The relation of response to $\mathrm{AUC}_{0-9}$, for those patients in whom the latter was determined, is shown in table 2 . The efficacy rate was $25 \%$ (5/20) for all patients with $\mathrm{AUC}_{0-9}$ levels of $\geqslant 10 \mathrm{ng} \cdot \mathrm{h} / \mathrm{ml}$. This efficacy rate increased to $57 \%(4 / 7)$ when data for only patients with $\mathrm{AUC}_{0-9}$ levels of $\geqslant 20 \mathrm{ng} . \mathrm{h} / \mathrm{ml}$ were analysed.

\section{NON-RESPONDERS}

In patients in whom atrioventricular re-entrant tachycardia could be reinduced, the change in tachycardia cycle length was measured at each of the five doses (table 3). The greatest increase in cycle length was seen at the $9 \mu \mathrm{g} / \mathrm{kg}$ dose (mean (SEM): 47 (12) ms). Though sample sizes were small, increases in cycle length appeared to be dose dependent in all but the highest dose group $(15 \mu \mathrm{g} / \mathrm{kg})$.

\section{REFRACTORY PERIODS}

Atrial and ventricular effective refractory periods were measured, whenever possible, at basic cycle lengths of 400 and $600 \mathrm{~ms}$. The baseline values and the changes in the refractory periods (table 4) show that dofetilide increased the effective refractory period in both atrium and ventricle; however, a dose-response relation was not clearly shown. No clear differences were seen between the increase in effective refractory period for responders versus nonresponders. As a result of the variability in the relatively small sample size, no formal statistical analysis was performed. 
The antegrade refractory period of the accessory pathway could be measured in 23 patients and the retrograde refractory period in 16. As with the atrial and ventricular refractory periods, the effective refractory period of the accessory pathway was consistently increased, whether measured antegradely or retrogradely (table 4). Because of the small number of patients and the difficulty in obtaining refractory period measurements for the accessory pathway in all five dosage groups, a clear doseresponse relation could not be demonstrated. However, when individual patient data were examined, non-inducibility appeared to be linked to notable prolongation of the effective refractory periods in most responders.

In one patient who developed atrial fibrillation before and during the dofetilide infusion $(1.5 \mu \mathrm{g} / \mathrm{kg})$, the minimum $\mathrm{RR}$ interval increased from 230 to $235 \mathrm{~ms}$ during the dofetilide infusion.

\section{SIDE EFFECTS}

Four patients reported side effects that were considered possibly related to dofetilide treatment. One patient experienced episodes of junctional rhythm and bundle branch block; one patient developed spontaneous atrioventricular re-entrant tachycardia identical to that previously induced in the electrophysiology laboratory; and one patient developed a short lasting episode of hypotension and hypoxia. The only serious side effect was reported in one patient who developed extrasystoles which degenerated into a new sustained, incessant, supraventricular tachycardia. Treatment was discontinued, and a paced ventricular beat terminated the tachyarrhythmia. No clinically relevant aberrations in haemodynamic or laboratory data were detected in the remaining patients. Four other patients developed transient QTc prolongations of $>600 \mathrm{~ms}$; two of these patients had received $15 \mu \mathrm{g} / \mathrm{kg}$ of dofetilide and the other two had received 1.5 and $6 \mu \mathrm{g} / \mathrm{kg}$ of dofetilide, respectively. Clinical resolution was seen for all treatment related side effects without subsequent adverse consequences.

\section{Discussion}

The main finding of this study is that intravenous dofetilide is a safe and effective agent for preventing the induction of atrioventricular re-entrant tachycardia. A clear and significant relation between dofetilide dose and antiarrhythmic response was demonstrated, as measured by the inability to reinduce the tachycardia. A minimum dose of $6 \mu \mathrm{g} / \mathrm{kg}$ was necessary for antiarrhythmic efficacy. A relation between plasma concentrations of dofetilide and efficacy was also demonstrated, as shown by a $25 \%$ efficacy rate when dofetilide AUC $_{0-9}$ was $\geqslant 10 \mathrm{ng} \cdot \mathrm{h} / \mathrm{ml}$ and a $57 \%$ efficacy rate when it was $\geqslant 20 \mathrm{ng} . \mathrm{h} / \mathrm{ml}$. These findings are consistent with the recommendations of Lévy, who-using a Sicilian Gambit ${ }^{18}$ approach - suggested targeting the potassium current with a class III agent in orthodromic atrioventricular re-entrant tachycardia. ${ }^{19}$
Intravenous dofetilide was safe in this patient population. Although four patients developed pronounced QT prolongation, none developed torsade de pointes. As periods of sinus rhythm alternated with paced periods during the electrophysiology study, and as the QT prolongation persisted beyond the completion of the electrophysiology study, it is unlikely that the absence of torsade de pointes was related to the ability to pace during the study.

This investigation is the first to measure dofetilide induced prolongation of both antegrade and retrograde refractoriness in accessory pathways in patients with atrioventricular re-entrant tachycardia. The mechanism of this type of tachycardia is believed to be circus movement re-entry involving the atrioventricular node and an accessory pathway. Whether the tachycardia is orthodromic or antidromic, a critical region in the re-entrant pathway of prolonged refractoriness defines the excitable gap of the circuit and is the target of antiarrhythmic drug action. A drug that increases refractoriness to a sufficient degree in this critical portion of the re-entrant circuit may terminate re-entry by effectively reducing the excitable gap to zero. ${ }^{20}$ Because we examined the efficacy of dofetilide at five separate doses, there were insufficient refractory period determinations to perform statistical analyses or draw strong conclusions about the site of action. However, based on the limited refractory results presented here and the very specific class III antiarrhythmic action of dofetilide, it seems probable that when the dose of dofetilide is sufficiently large the drug prolongs the refractoriness of a critical site. Given the lack of troublesome side effects of dofetilide, even at the highest dose, it may be a useful treatment for preventing atrioventricular re-entrant tachycardia.

Dofetilide can be used effectively to treat other re-entrant supraventricular arrhythmias, atrial fibrillation, and atrial flutter, ${ }^{14-16} 2122$ and it compares favourably with class IC antiarrhythmic agents. ${ }^{23}$ The majority of these arrhythmias are caused by circus movement re-entry, in which dofetilide can similarly increase the probability of conduction block at the critical site within re-entrant pathways. Thus the ability of dofetilide to prevent or terminate supraventricular re-entrant arrhythmias is linked to its ability to prolong refractoriness, as reflected in the surface ECG QT interval.

\section{COMPARISON WITH OTHER ANTIARRHYTHMIC} DRUGS

The efficacy of dofetilide in preventing the induction of atrioventricular re-entrant tachycardia is similar to that of other drugs used to treat this arrhythmia: $31 \%$ for flecainide, ${ }^{24}$ $31-50 \%$ for propafenone, ${ }^{24-26} 30 \%$ for amiodarone, ${ }^{27}$ and $33 \%$ for sotalol. ${ }^{26}$ However, these other drugs-along with additional agents suggested for the treatment of atrioventricular re-entrant tachycardia-have significant side effects. Class I agents are proarrhythmic and have negative inotropic and negative dromotropic effects. ${ }^{8}$ The class III agent sotalol produces hypotension and has significant $\beta$ 
blocking effects. ${ }^{3}$ Amiodarone, another class III agent, is effective but has serious systemic side effects. ${ }^{7}$ Like digoxin, the class IV agent verapamil can shorten the antegrade refractory period of the accessory pathway, leading to a deleterious increase in ventricular rate and ventricular fibrillation. ${ }^{8}$

The efficacy and safety of intravenous dofetilide found in this study of patients with atrioventricular re-entrant tachycardia suggest that an oral form of the drug should be evaluated for the long term suppression of this type of arrhythmia. Further studies using oral dofetilide need to be performed to determine its exact role in the treatment of these patients.

CONCLUSIONS

Dofetilide was effective in preventing atrioventricular re-entrant tachycardia in patients with inducible arrhythmia. The potent and selective $\mathrm{I}_{\mathrm{Kr}}$ blocking effect, combined with its side effect profile, qualifies this agent as a prime candidate for the treatment of patients with atrioventricular re-entrant tachycardia. The results presented here point to the need for additional clinical studies to determine the efficacy of oral dofetilide for the long term prevention of this arrhythmia.

This study was supported by a grant from Pfizer Central Research, UK.

1 Echt DS, Liebson PR, Mitchell LB, et al. Mortality and morbidity in patients receiving encainide, flecainide, or plamorbidity in patients receiving encainide, flecainide, or plaMed 1991;324:781-8.

2 Jordaens L, Gorgels A, Stroobandt R, et al. Efficacy and safety of intravenous sotalol for termination of paroxysmal supraventricular tachycardia. Am f Cardiol 1991;68:35-40.

3 Sung RJ, Tan HL, Karagounis L, et al. Intravenous sotalol for the termination of supraventricular tachycardia and atrial fibrillation and flutter: a multicenter, randomized, double-blind, placebo-controlled study. Am Heart $\mathcal{f} 1995$ 129:739-48.

4 Millar RNS. Efficacy of sotalol in controlling reentrant supraventricular tachycardias. Cardiovasc Drugs Ther 1990 4:625-9.

5 Amiodarone Trials Meta-Analysis Investigators. Effect of prophylactic amiodarone on mortality after acute myocardial infarction and in congestive heart failure: meta-analysis of individual data from 6500 patients in randomised trials. Lancet 1997;350:1417-24.

6 Chun SH, Sager PT, Stevenson WG, et al. Long-term Chun SH, Sager PT, Stevenson WG, et al. Long-term efficacy of amiodarone for the maintenance of normal sinus
rhythm in patients with refractory atrial fibrillation or flutrhythm in patients with refractory

7 Vorperian VR, Havighurst TC, Miller S, et al. Adverse effects of low dose amiodarone: a meta-analysis. $\mathcal{F} \mathrm{Am} \mathrm{Coll}$ Cardiol 1997;30:791-8.
8 Goy J-J, Fromer M. Antiarrhythmic treatment of atrioventricular tachycardias. $\mathcal{f}$ Cardiovasc Pharmacol 1991; tricular tachycardias.

9 Carmeliet E. Voltage- and time-dependent block of the delayed $\mathrm{K}^{+}$current in cardiac myocytes by dofetilide. $\mathcal{F}$ Pharmacol Exp Ther 1992;262:809-17.

10 Gwilt M, Arrowsmith JE, Blackburn KJ, et al. UK-68,798: a novel, potent and highly selective class III antiarrhythmic agent which blocks potassium channels in cardiac cells. $\mathcal{F}$ Pharmacol Exp Ther 1991;256:318-24.

11 Sedgwick ML, Dalrymple I, Rae AP, et al. Effects of the new class III antiarrhythmic drug dofetilide on the atrial and ventricular intracardiac monophasic action potential in patients with angina pectoris. Eur Heart $\mathcal{f} 1995 ; 16: 1641-6$.

12 Echt DS, Lee JT, Murray KT, et al. A randomized, doubleblind, placebo-controlled, dose-ranging study of dofetilide in patients with inducible sustained ventricular tachyarin patients with inducible sustained ventricular tachya

13 Sedgwick ML, Rasmussen HS, Cobbe SM. Clinical and electrophysiologic effects of intravenous dofetilide (UK68,798), a new class III antiarrhythmic drug, in patients $68,798)$, a new class III antiarrhythmic drug, in
with angina pectoris. Am 7 Cardiol 1992;69:513-7.

14 Falk RH, Pollak A, Singh SN, et al. Intravenous dofetilide, a class III antiarrhythmic agent, for the termination of sustained atrial fibrillation or flutter. $\mathcal{F} \mathrm{Am}$ Coll Cardiol 1997;29:385-90. 15 Greenbaum R, Campbell TJ, Channer KS, et al. Conversion
of AF and maintenance of sinus rhythm by dofetilide abstract P3682]. Eur Heart f 1998;19(suppl):661.

16 Singh SN, Berk MR, Yellen LG, et al. Efficacy and safety of oral dofetilide in maintaining normal sinus in patients with 2145]. Circulation 1997;96(suppl 8):I-383.

17 Walker DK, Aherne GW, Arrowsmith JE, et al. Measurement of the class III antidysrhythmic drug, UK-68,798, in ment of the class II antidysrhythmic drug, UK-68,798, in 9:141-9.

18 Task force of the Working Group on Arrhythmias of the European Society of Cardiology. The Sicilian Gambit. A new approach to the classification of antiarrhythmic drugs based on their actions on arrhythmogenic mechanisms. Circulation 1991;84:1831-51.

19 Lévy S, Ricard P. Using the right drug: a treatment algorithm for regular supraventricular tachycardias. Eur Heart 7 1997; 18: C27-32.

20 Wit AL, Coromilas J. Role of alterations in refractoriness and conduction in the genesis of reentrant arrhythmias. Implications for antiarrhythmic effects of class III drugs. Am 7 Cardiol 1993;72:3-12F.

21 Sedgwick ML, Lip G, Rae AP, et al. Chemical cardioversion of atrial fibrillation with intravenous dofetilide. Int $\mathcal{F}$ Cardiol 1995;49:159-66.

22 Suttorp MJ, Polak PE, van 't Hof A, et al. Efficacy and safety of a new selective class III antiarrhythmic agent dofetilide in paroxysmal atrial fibrillation or atrial flutter. Am 7 Cardiol 1992;69:417-19.

23 Crijns HJGM, Van Gelder IC, Kingma JH, et al. Atrial flutter can be terminated by a class III antiarrhythmic drug but not by a class IC drug. Eur Heart f 1994;15:1403-8.

24 O’Nunain S, Garratt CJ, Linker NJ, et al. A comparison of intravenous propafenone and flecainide in the treatment of tachycardias associated with the Wolff-Parkinson-White syndrome. Pacing Clin Electrophysiol 1991;14:2028-34.

25 Chen X, Borggrefe M, Martinez-Rubio A, et al. Efficacy of ajmaline and propafenone in patients with accessory pathways: a prospective randomized study. $\mathcal{F}$ Cardiovasc Pharmacol 1994;24:664-9.

26 Manz M, Lüderitz B. Supraventricular tachycardia and preexcitation syndromes: pharmacological therapy. Eur Heart 7 1993;14:91-8.

27 Wellens HJJ, Brugada P, Abdollah H. Effect of amiodarone in paroxysmal supraventricular tachycardia with or without Wolff-Parkinson-White syndrome. Am Heart $\mathcal{F}$ 1983;106: 876-80. 